\title{
BRACKISH RESOURCES AQUIFER CHARACTERIZATION SYSTEM (BRACS) FOR TEXAS GROUNDWATER
}

\author{
Andrea Croskrey \\ 2017 GSA South-Central Section Meeting \\ T6. "Karst: From Sinkholes to Springs and Everything in Between" \\ Monday March 13th \\ Omni Colonnade San Antonio, Grand Ballroom B
}


The following presentation is based upon professional research and analysis within the scope of the Texas Water Development Board's statutory responsibilities and priorities but, unless specifically noted, does not necessarily reflect official Board positions or decisions. 


\section{Primary Responsibilities:}

- State Water Plan

- Funding

- Water Resource Data

- Outreach

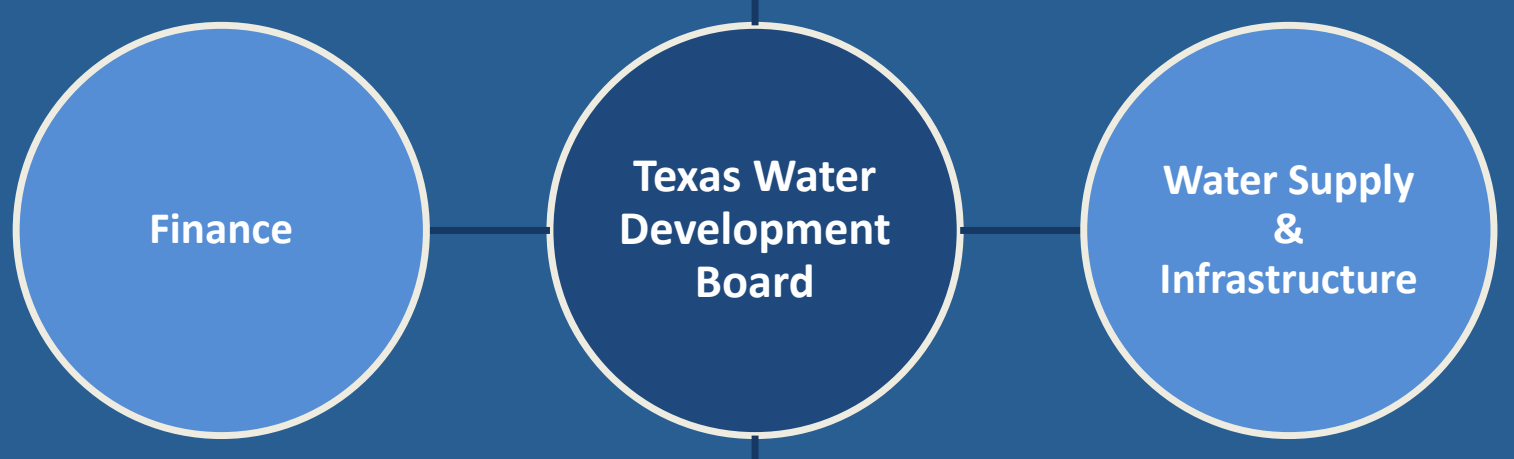

"To provide leadership, information, education, and support for planning, financial assistance, and outreach for the conservation and responsible development of water for Texas"

Water Science

$\&$

Conservation
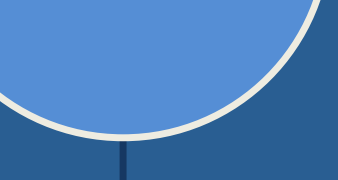

Operations \& Administration www.twdb.texas.gov

f www.facebook.com/twdboard @twdb
Texas Water

Development Board 


\section{Innovative Water Technologies}

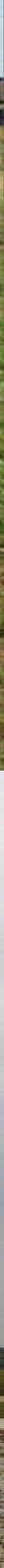




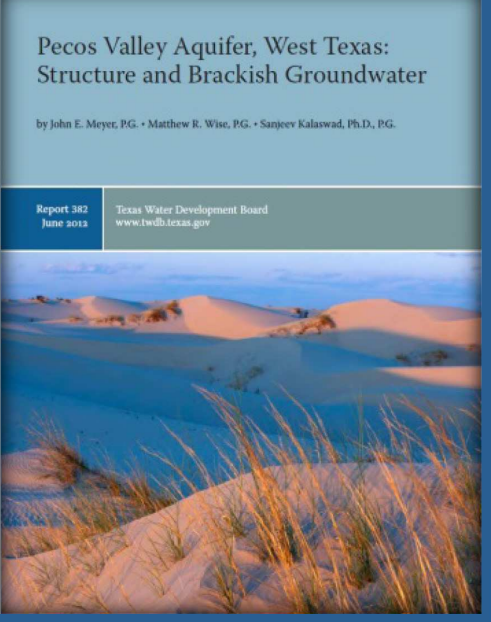

Geologic Characterization of and Data Collection in the Corpus Christi Aquifer

Storage and Recovery Conservation District

and Surrounding Counties

open File Report i2-DI

September 2012

John E. Meger, P.G.

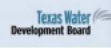

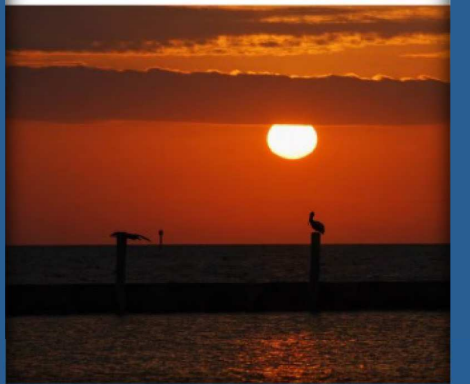

Brackish Resources Aquifer

Characterization System Database

Data Dictionary

Open File Reporr 12-202. Second Edtition

Seprember 2014

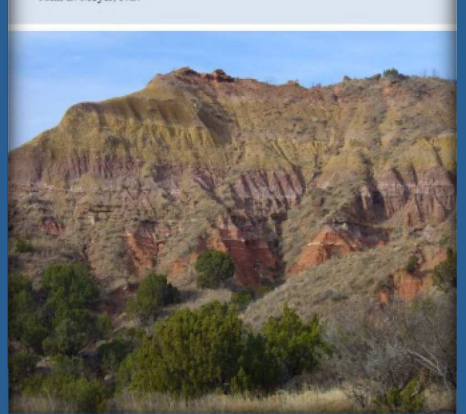

Queen City and Sparta Aquifers,

Atascosa and McMullen Counties, Texa:

Structure and Brackish Groundwater

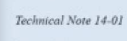

May 2014
Marticew R Wise, PC.

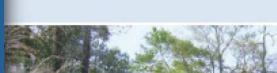

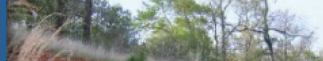

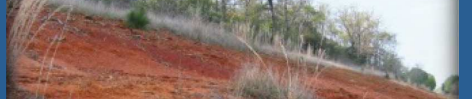
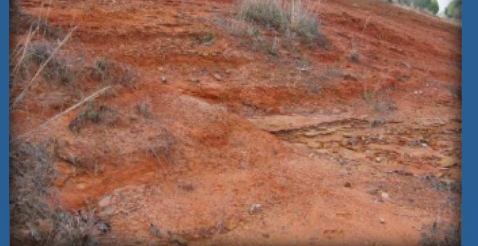

Brackish Groundwater in the Gulf Coast Aquifer, Lower Rio Grande Valley, Texas

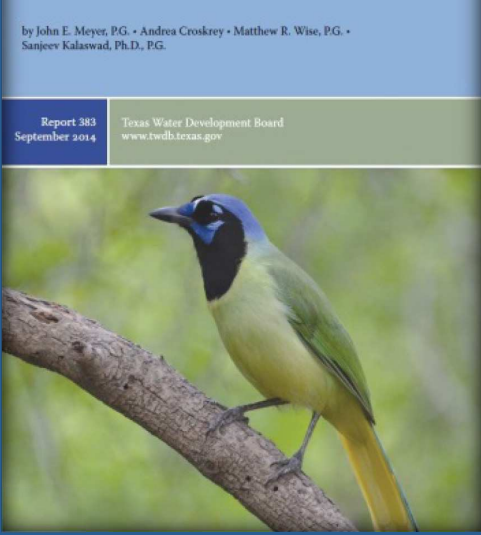

\section{Brackish \\ Resources \\ Aquifer Characterization System (BRACS)}

- TWDB program since 2009

- Mapping brackish aquifers

- Knowledge gap

- Legislation

www.twdb.texas.gov

f www.facebook.com/twdboard @twdb
Texas Water

Development Board 


\section{House Bill 30 \\ (84th Texas Legislature, 2015)}

\section{Directed TWDB to:}

- define brackish groundwater production zones

- estimate productivity over 30 \& 50 year periods

- recommend groundwater monitoring

- four aquifers due December, 2016

- all aquifer studies due December, 2022 


\section{Brackish Groundwater}

\section{Saltier than fresh water, less salty than seawater}

\begin{tabular}{|c|c|c|}
\hline Groundwater Salinity Classification & Salinity Zone Code & $\begin{array}{r}\text { Total Dissolved Solids Concentration } \\
\text { (units: milligrams per liter) }\end{array}$ \\
\hline Fresh & FR & 0 to 1,000 \\
\hline Slightly Saline & SS & 1,000 to 3,000 \\
\hline Moderately Saline & MS & 3,000 to 10,000 \\
\hline Very Saline & VS & 10,000 to 35,000 \\
\hline Brine & BR & Greater than 35,000 \\
\hline
\end{tabular}

Drinking Water Limit

Major/Minor Aquifer Mapped Limit

Seawater

\section{Groundwater Salinity Classification}

Source: modified from Winslow and Kister, 1956

www.twdb.texas.gov

f www.facebook.com/twdboard @twdb 


\title{
BRACS Program
}

\author{
Brackish \\ Resources \\ Aquifer \\ Characterization \\ System
}

Current studies and projects

$\checkmark 2$ Bm. Blossom Aquifer (Contract No. 1600011951)

$\square$ Lp. Lipan Aquifer

Nh. Nacatoch Aquifer (Contract No. 1600011952)

Ty. Trinity Aquifer (Contract No. 1600011950)

UCP. Upper Coastal Plain Aquifers

\section{Completed studies and projects}

Be. Blaine Aquifer (Contract No. 1600011948)

Cz. Carrizo-Wilcox Aquifer (Contract No. 1548301855)

GC1. Gulf Coast Aquifer (Report 12-01)

GC2. Gulf Coast Aquifer (Report 383)

GC3. Gulf Coast Aquifer (Contract No. 1600011947)

PV. Pecos Valley Aquifer (Report 382)

QS. Queen City-Sparta Aquifer (Report 14-01)

Rr. Rustler Aquifer (Contract No. 1600011949)

Proposed studies are conceptual and may or may not

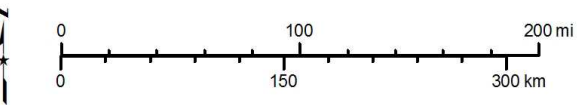

$200 \mathrm{mi}$ ,

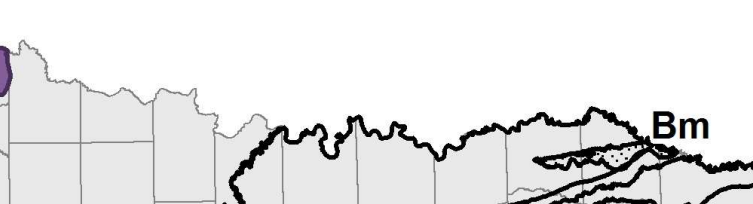




\section{General Methodology}

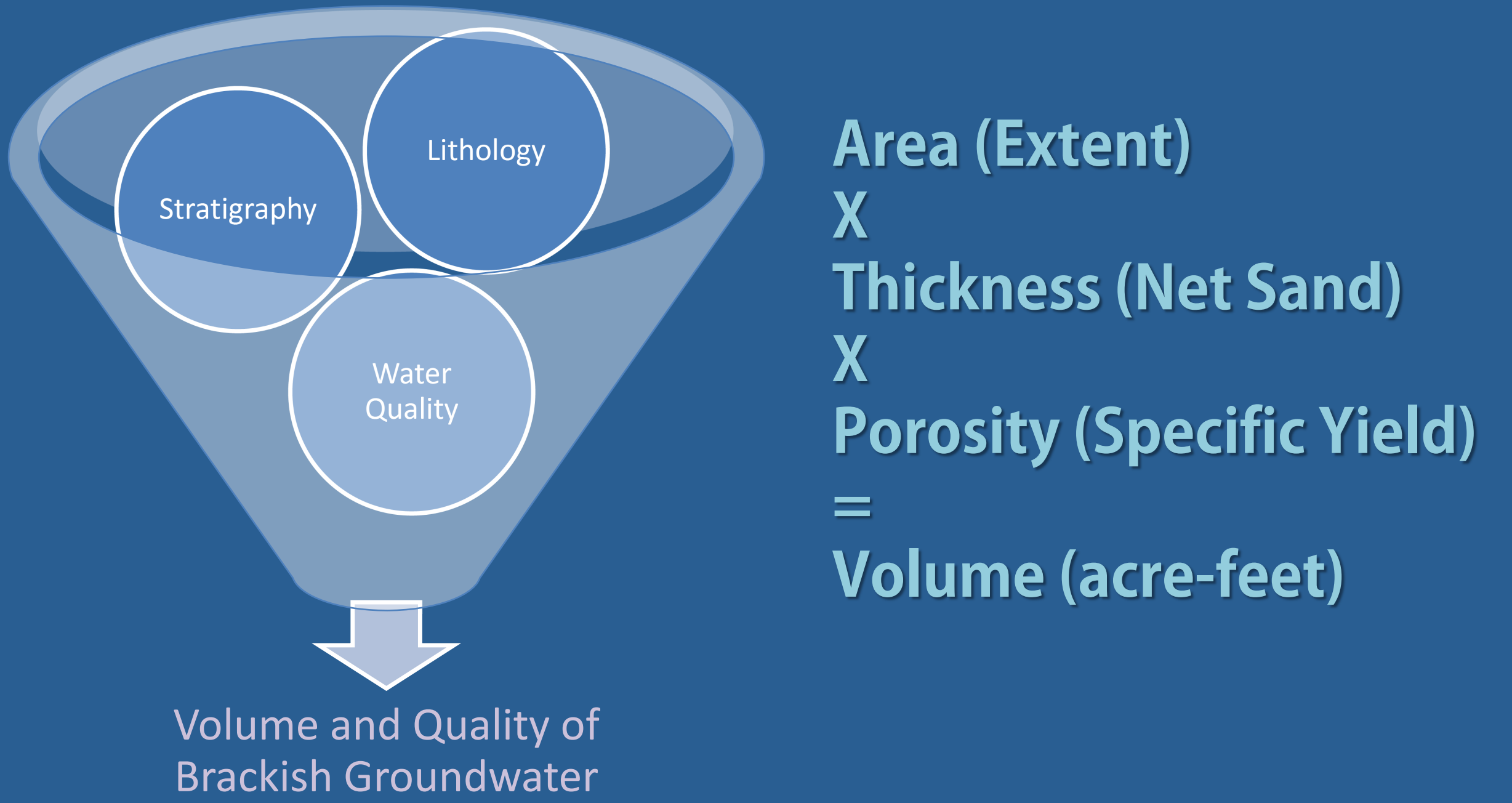

www.twdb.texas.gov

f www.facebook.com/twdboard $Y$ @twdb
Texas Water

9
Development Board 


\section{Log analysis: Stratigraphy and Lithology}

BRACS Well ID 42889
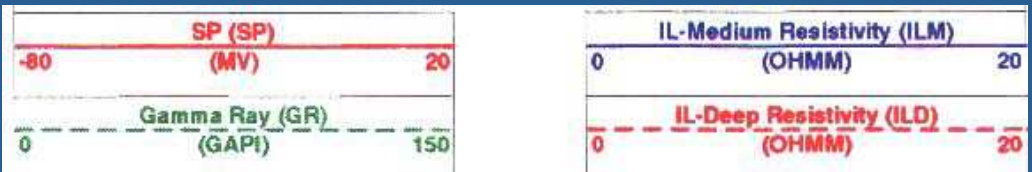

Beaumont Fm

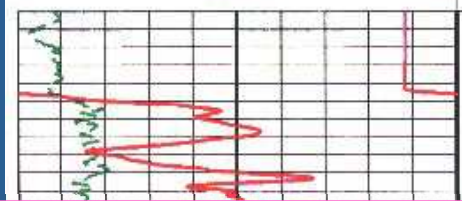

Lissie Fm

Willis Fm

Upper Goliad Fm

Upper Goliad Fm
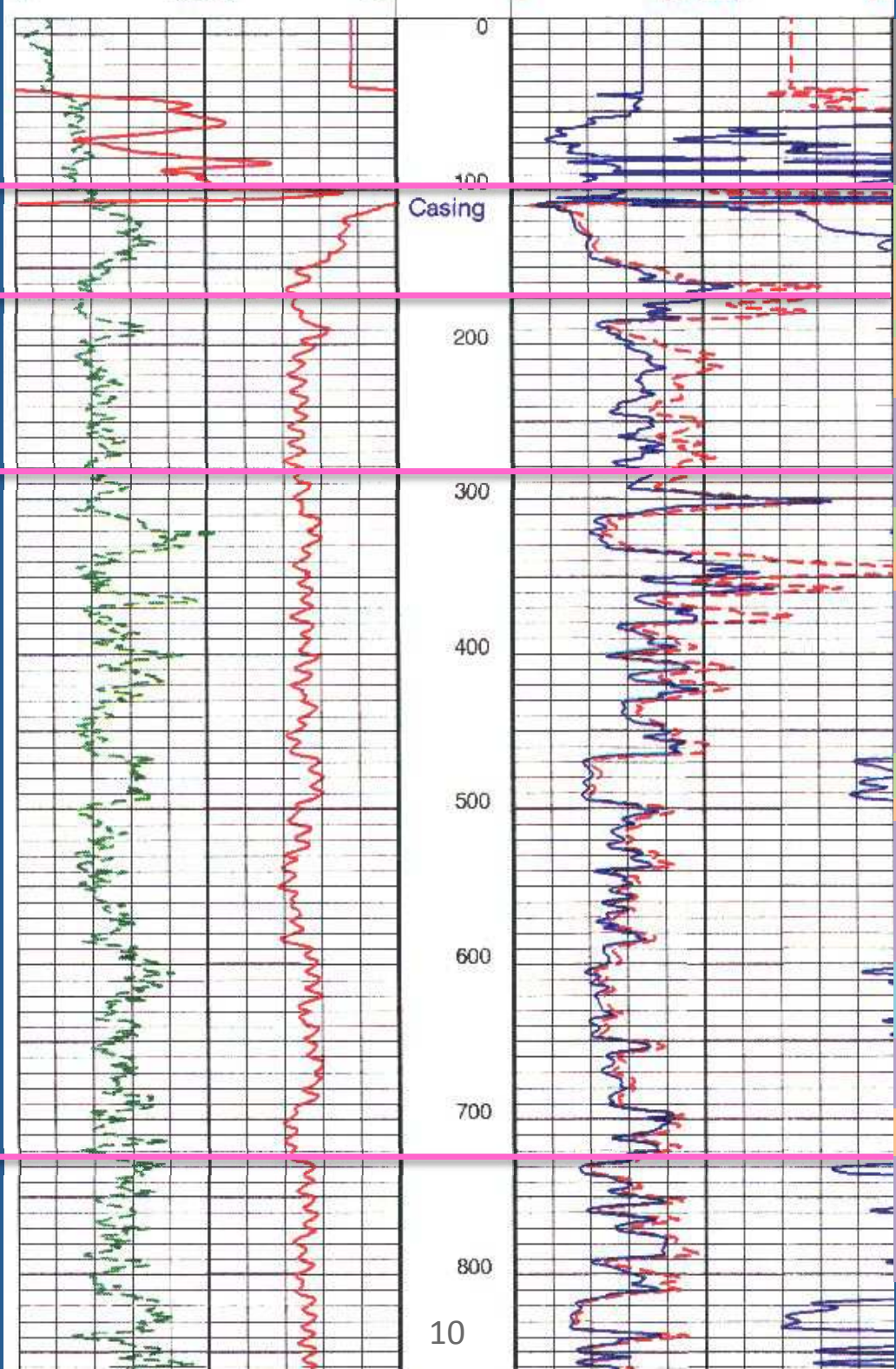

no data

clay

sand

sand

sand with clay

sand with clay

sand with clay

clay with sand

sand with clay sand

sand with clay 


\section{BRACS Database: Primary Tables}

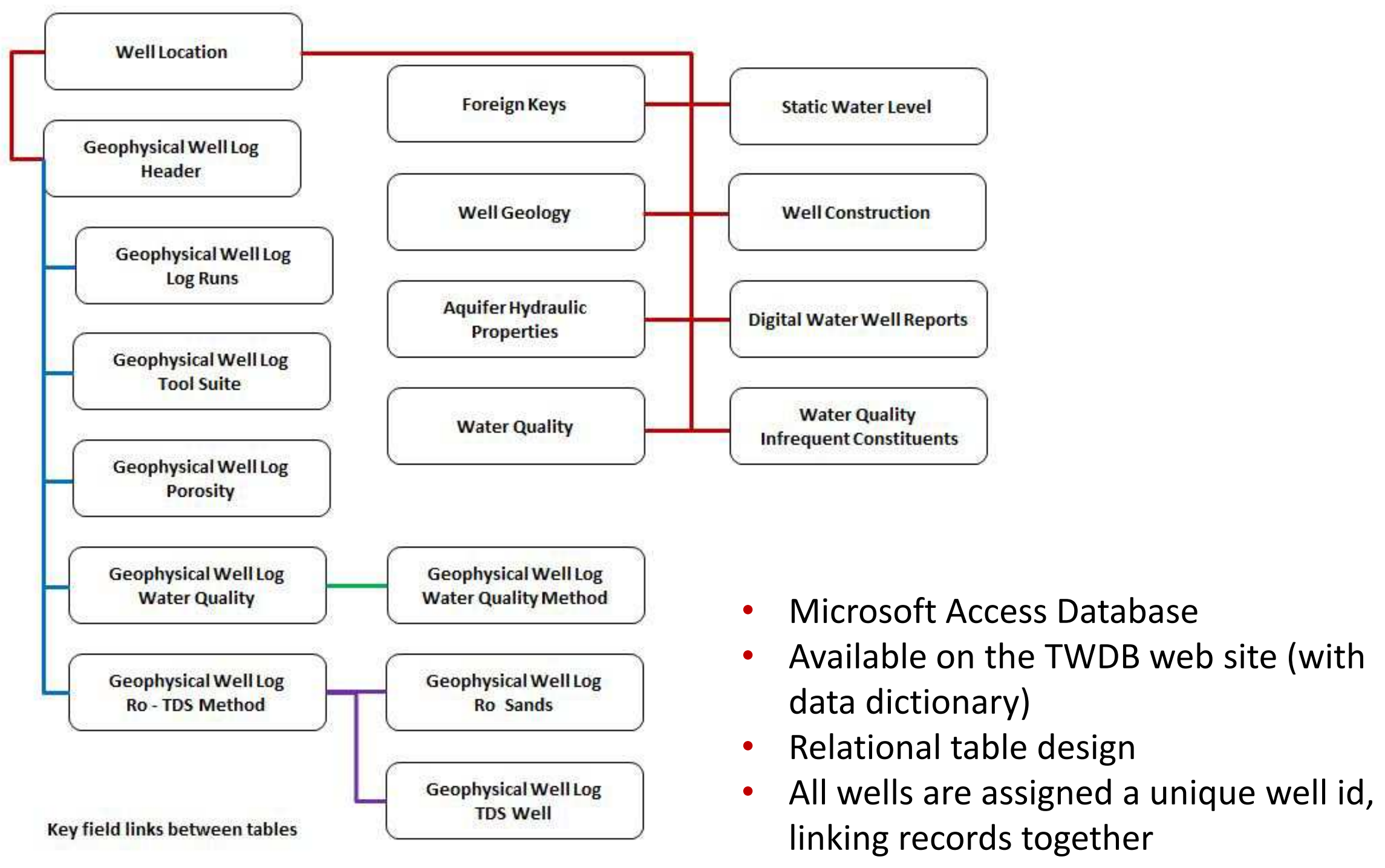

Well_ID

Well_ID, GL_Number

Well_Id, GL_Number, DF

Well_ID, GL_Number, Ro_TDS_Number 


\section{BRACS Database: Water Quality Log Analysis Calculations}

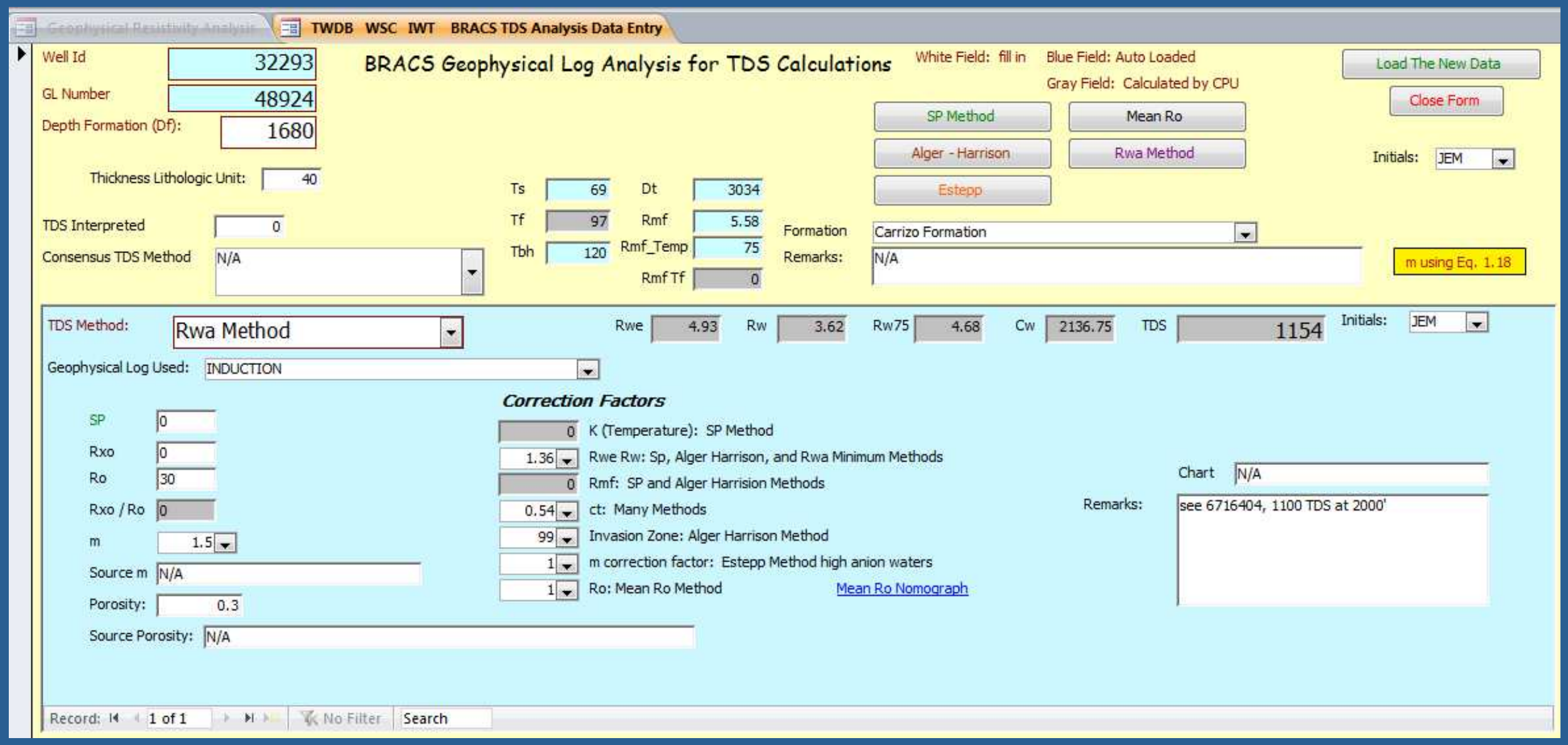

www.twdb.texas.gov

f www.facebook.com/twdboard @twdb
Texas Water Development Board 


\section{GIS: Interpolate Points to make Rasters}

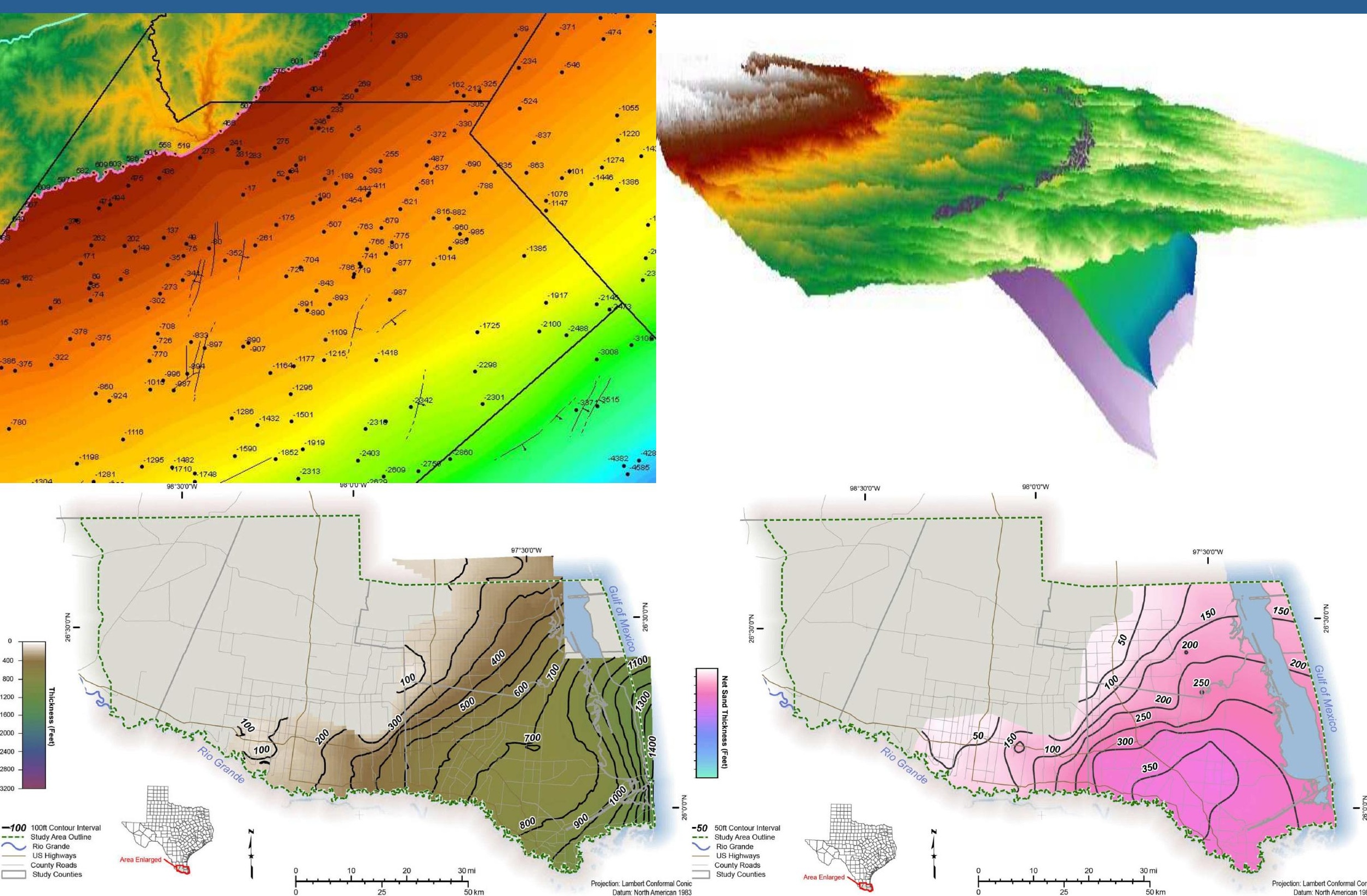




\section{Download Our Database!}

http://www.twdb.texas.gov/innovativewater/bracs/database.asp

The 1 Brackish Resources Aquifer Characterization System (BRACS)

Database was designed to store well and geology information in support of projects to characterize the brackish groundwater resources of Texas. The BRACS database is fully relational, with self-documenting object naming. The database design relies on extensive use of lookup tables. The BRACS database is a Microsoft Access 2007 format that has been compressed with the WinZip utility. This database will be updated periodically; the date of the last update is embedded in the filename.

This database was developed for use by TWDB staff in support of the BRACS program. The information changes on a daily basis and users should read the disclaimer below. If you have any questions, please contact John Meyer at 512-463-8010.

A data dictionary to accompany the BRACS Database is now available for download. The dictionary describes each primary table in the database and custom tables developed for a study.

Brackish Resources Aquifer Charaterization System Database Data Dictionary, Second Edition, TWDB Open File Report 12-02, September 2014 (3 MB)
Brackish Resources Aquifer Characterization System Database Data Dictionary

Open File Repart 12-02, Second Editiow

September 2014

John E. Meyer, P.G

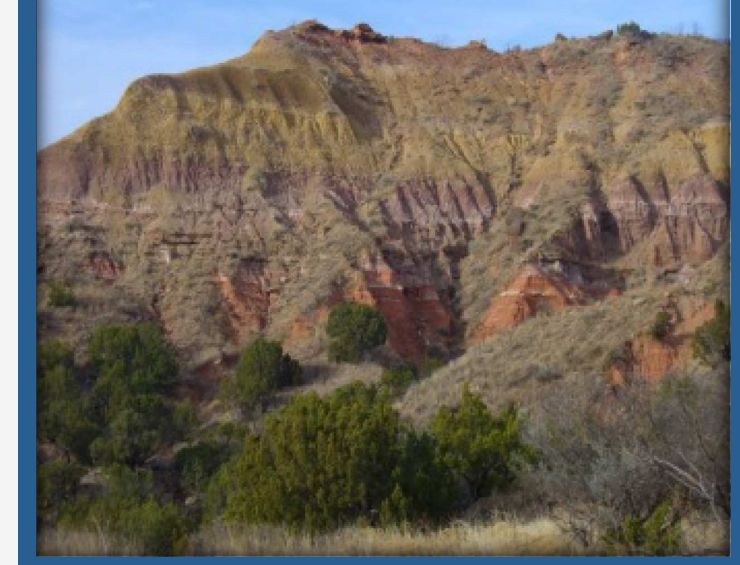

www.twdb.texas.gov

www.facebook.com/twdboard @twdb
Texas Water Development Board 


\section{Download Geophysical Well Logs!}

1. Download logs on a per well basis using Water Data Interactive website https://www2.twdb.texas.gov/apps/waterdatainteractive/groundwaterdataviewer

WUTER DATA Interactive

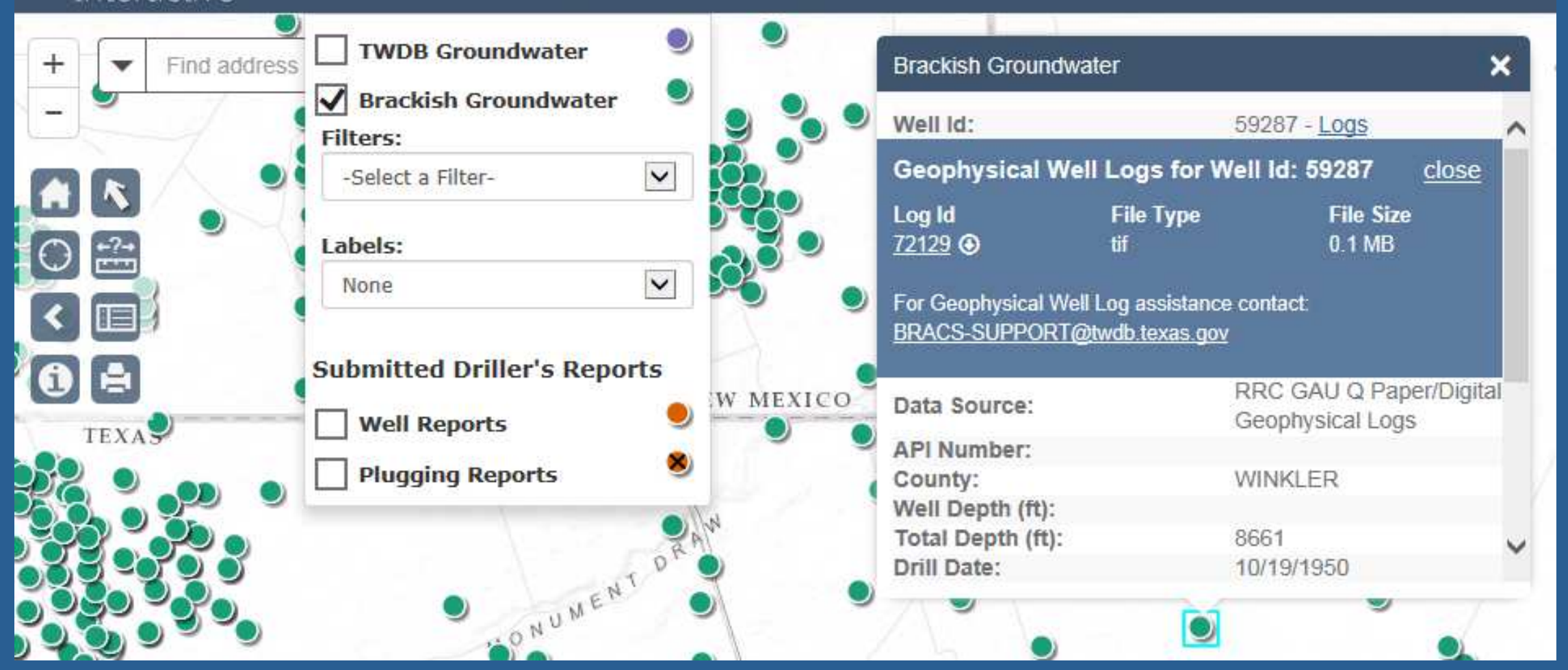

2. Instructions for requesting a large volume of logs on a county basis http://www.twdb.texas.gov/innovativewater/bracs/WellLogs.asp 


\section{Studies and Contracted Projects}

Completed Studies

http://www.twdb.texas.gov/innovativewater/bracs/studies.asp

\section{Completed Studies}

\begin{tabular}{|c|c|c|c|}
\hline $\begin{array}{l}\text { Complete } \\
\text { Date }\end{array}$ & Project & $\begin{array}{l}\text { Report } \\
\text { Number }\end{array}$ & Funding \\
\hline \multirow[t]{2}{*}{$09 / 2014$} & $\begin{array}{l}\text { Brackish Groundwater in the Gulf Coast } \\
\text { Aquifer, Lower Rio Grande Valley, } \\
\text { Texas }\end{array}$ & 383 & In-house \\
\hline & $\begin{array}{l}\text { Gulf Coast Aquifer GIS Datasets } \\
(128.0 \mathrm{MB})\end{array}$ & & \\
\hline
\end{tabular}

Current and Completed Contracted Projects

http://www.twdb.texas.gov/innovativewater/bracs/projects.asp

$11 / 2016$
Identification of

Potential Brackish

Groundwater

Production Areas -

Rustler Aquifer
1600011949 INTERA, Inc. 


\section{We need your data!}

Andrea Croskrey

Geologist

Innovative Water Technologies

Texas Water Development Board

andrea.croskrey@twdb.texas.gov

(512) 463-2865

http://www.twdb.texas.gov/innovativewater/index.asp

Interactive 2017 Water Plan:

https://2017.texasstatewaterplan.org/statewide 